\title{
Structural transitions and mechanochemical coupling in the nucleoprotein filament explain homology selectivity and Rad51 protein cooperativity in cellular DNA repair
}

\author{
Scott X. Atwell, ${ }^{1}$ Daniel Migliozzi ${ }^{1,2}$ Aurélie Dupont, ${ }^{1,3}$ Jean-Louis Viovy, ${ }^{4}$ and Giovanni Cappello ${ }^{1,3, *}$ \\ ${ }^{1}$ Institut Curie, PSL Research University, Centre National de la Recherche Scientifique, Sorbonne Universités, UPMC Univ Paris 06, Unité \\ Mixte de Recherche 168, 75005 Paris, France \\ ${ }^{2}$ Laboratory of Microsystems, Ecole Polytechnique Fédérale de Lausanne, Lausanne 1015, Switzerland \\ ${ }^{3}$ Université Grenoble Alpes, Laboratoire Interdisciplinaire de Physique, CNRS, F-38000 Grenoble, France \\ ${ }^{4}$ Institut Curie, PSL Research University, Centre National de la Recherche Scientifique, Sorbonne Universités, UPMC Univ Paris 06, Unité \\ Mixte de Recherche 168, Institut Pierre Gilles de Gennes, MMBM Group, 75005 Paris, France
}

(Received 23 October 2019; accepted 17 February 2020; published 12 March 2020)

\begin{abstract}
The nucleoprotein filament (NPF) is the fundamental element of homologous recombination (HR), a major mechanism for the repair of double-strand DNA breaks in the cell. The NPF is made of the damaged DNA strand surrounded by recombinase proteins, and its sensitivity to base-pairing mismatches is a crucial feature that guarantees the fidelity of the repair. The concurrent recombinases are also essential for several steps of HR. In this work, we used torque-sensitive magnetic tweezers to probe and apply mechanical constraints to single nucleoprotein filaments (NPFs). We demonstrated that the NPF undergoes structural transitions from a stretched to a compact state, and we measured the corresponding mechanochemical signatures. Using an active two-state model, we proposed a free-energy landscape for the NPF transition. Using this quantitative model, we explained both how the sensitivity of the NPF to the homology length is regulated by its structural transition and how the cooperativity of Rad51 favors selectivity to relatively long homologous sequences.
\end{abstract}

DOI: 10.1103/PhysRevE.101.032407

\section{INTRODUCTION}

Homologous recombination (HR) is the process that cells employ to faithfully repair double-strand damages of the DNA. To reconstitute the genetic information lost in the damaged DNA, HR uses as template the homologous sequence stored in the sister chromatid [1-3]. HR is thus essential for the maintenance of genome integrity and to avoid cancer development.

In humans, HR is mediated by the protein Rad51. In the presence of adenosine triphosphate (ATP), the Rad51 protein assembles on a single-strand portion of the damaged DNA fragment and forms a nucleoprotein filament (NPF). Then, the NPF gets in contact with the homologous double-stranded DNA to initiate the homology search. When, and only when, the NPF has found its homologous counterpart, a three-strand synapse is established and the strand exchange is initiated [4]. Although the strand exchange is irreversible and requires ATP hydrolysis [5], the search phase does not [6]. It is thus fully reversible and can be repeated indefinitely, until the homologous sequence is found.

In this article, we discuss the ability of the recombinase protein Rad51 to discriminate between homologous and nonhomologous sequences. Clearly, the free energy associated with Watson-Crick pairing between homologous sequences drives the homology recognition. Recent works have provided a precise experimental determination of the free energy

*Giovanni.Cappello@univ-grenoble-alpes.fr reduction during the recombination-mediated base pairing [7] and evaluated the energy cost associated with a given mismatch [8]. Although the mismatch cost is small, it is sufficient to impede the recombination. The reasons for this sensitivity are still debated, but can be attributed to the way the Rad51 protein redefines the reaction path.

A peculiarity of the Rad51 protein is to modify the topological and mechanical state of the broken DNA fragment: polymerizing on the DNA fragment in a helical manner $[9,10]$, Rad51 stretches the DNA molecule to $150 \%$ and unwinds it by $43 \%$ with respect to the crystallographic conformation (supercoiling degree $\sigma=-0.43$ ). In this canonical conformation, the NPF stores a given amount of elastic energy. Thus, an effective energy barrier appears in the energy landscape of homologous recombination. We hypothesize that this barrier impedes the spontaneous recombination between similar, but nonhomologous, DNA sequences. In this work, we model and estimate this energy barrier, and we discuss the possible impacts of such a barrier on the specificity of homologous recombination.

Single-molecule experiments have enabled the direct observation of NPF dynamics [11-13] and provided the longitudinal and torsional stiffness of the NPF [14,15], two essential parameters to estimate the energy barrier. Together with electron microscopy, single-molecule experiments have also proven the existence of a compact NPF [9,16,17]. To draw the energy landscape of the NPF in interaction with the three DNA strands, we proposed an active two-state model. We introduced two major hypotheses: (i) the NPF transition from the stretched to the compact form $(S \rightarrow C)$ is induced by the 
torque developed during the interaction between the two DNA molecules; and (ii) the $S \rightarrow C$ transition is associated with ATP hydrolysis and, thus, makes the reaction irreversible. To verify the prediction of the active two-state model, we used hybrid magnetic tweezers to manipulate a single NPF and to measure the torque developed during the interaction between the Rad51 protein on the DNA molecule. We showed that this model introduces a dynamic instability, located at the critical energy that discriminates between homologous and nonhomologous sequences. Taken together, our experimental and modeling work identify a physical mechanism to account for the high sensitivity and reliability of HR.

\section{MATERIALS AND METHODS}

\section{A. Experimental setup}

The experimental setup used here was an improvement of the one proposed by Lipfert et al. [18]. It aims at easily switching between "classical" and "free-orbiting" [19] magnetic tweezers. The first are useful to impose the supercoiling degree, while the second ones allow a free rotation of the beads around the vertical axis. The setup is constituted by a main hollow cylindrical magnet [white magnet in Fig. 1(a), R06-02-02-G, Supermagnete] coupled with a pair of side cylindrical magnets (black magnets, S-04-07-N, Supermagnete). The cylinder imposes a vertical gradient of the magnetic field that depends on its distance from the bead. This gradient is used to pull the bead up with a defined force. The side magnets can be raised or lowered at will. When lowered, the smaller magnets add a horizontal component to the magnetic field that hinders rotations of the beads. If raised, the contribution of the smaller magnets is negligible and thus equivalent to freeorbiting magnetic tweezers. Translations and rotations of the magnet were performed with five independent stepper motors.

The torque was calibrated experimentally for each bead and at different heights of the lateral magnets. We recorded the angular fluctuations of the bead, and through Boltzmann's distribution of the fluctuation amplitude we deduced the potential energy of the bead with respect to its angular coordinate. For small angular fluctuations the potential is approximately harmonic, thus by fitting the power spectrum of the bead fluctuations to a Lorentzian function, we simultaneously evaluate the angular stiffness of the trap and the viscous drag of the bead.

\section{B. Synthesis of Rad51 and DNA}

Human Rad51 was expressed in Escherichia coli and then purified following the protocol described in Ref. [16]. Two different DNA constructs were used. The first one was composed of a 14 435-bp central fragment ligated at one end to a multidigoxigenin-labeled DNA fragment of $672 \mathrm{bp}$ and at the other end to a multibiotin-labeled fragment of $834 \pm 81$ bp. All fragments were obtained by polymerase chain reaction of the $\lambda$-phage DNA. The central fragment was amplified between the positions 22180 and 37096 (Expand Long Template PCR System, Roche) and digested by MluI and EagI (New England Biolabs) at, respectively, 22220 and 36654 . The biotin fragment was amplified between positions 35901 and 37568 with biotin-modified deoxyuridine triphosphate (dUTP, Roche) and digested by EagI at 36 554. The

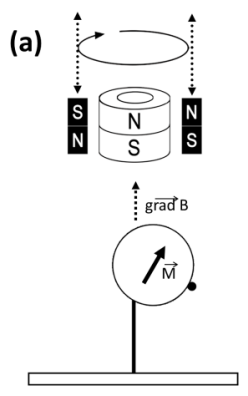

(b)

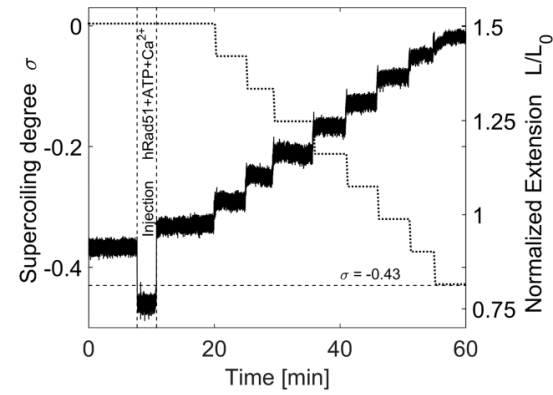

(c)

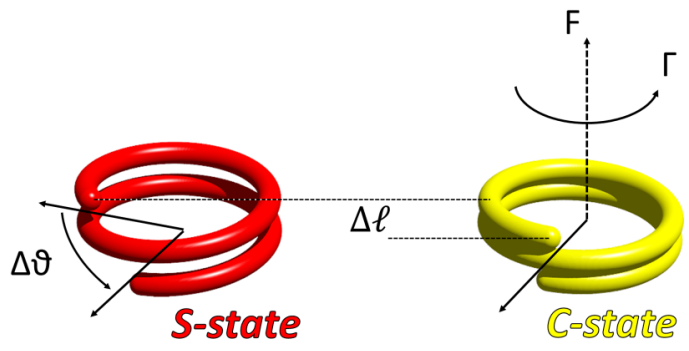

FIG. 1. (a) Schematic of the hybrid magnetic tweezers. Small magnets on both sides of the main hollow cylindrical magnet are mobile in the vertical direction and able to rotate around the central axis. A single NPF is bound to a glass slide on one extremity and to a magnetic bead on the other. The magnetization moment of the bead aligns with the quasivertical magnetic field which also has a small horizontal component. The field gradient remains vertical. (b) Supercoiling degree (dotted line) and normalized extension (solid line) of a NPF during controlled unwinding with the HMT. The dashed line indicates the supercoiling degree of the canonical form of the NPF. $\sigma=-0.43$. (c) A schematic view of the two-state model of the Rad51 protein in interaction with the DNA molecule (nucleoprotein filament). The transition from the $S$ state to the $C$ state $(S \rightarrow C)$ requires a positive twist $(\Delta \vartheta)$ and compression $(\Delta \ell)$. Whereas the hybrid magnetic tweezers always apply a tension $(F \geqslant 0)$, they allow both positive and negative torques $(\Gamma)$ to facilitate the $S \rightleftharpoons C$ transition bidirectionally.

digoxigenin fragment was amplified between positions 20281 and 20962 with digoxigenin-modified dUTP (Roche) and digested by MluI at 20952 . The second DNA construct was composed of a 10338 -bp central fragment ligated at one end to a multidigoxigenin-labeled DNA fragment of $907 \pm 172 \mathrm{bp}$ and at the other end to a multibiotin-labeled fragment of $696 \pm 496 \mathrm{bp}$. The central fragment was obtained by transformation of the pREP4 vector in E. coli cells (Turbo Competent E. coli cells, New England Biolabs) which was linearized through digestion by HindIII and NotI (New England Biolabs) at positions 592 and 603, respectively. The digoxigenin and biotin fragments were obtained by polymerase chain reaction (PCR) with digoxigenin-modified or biotin-modified dUTP (Roche). The digoxigenin fragment was amplified between positions 43063 and 44875 of the $\lambda$-phage DNA and digested by HindIII at 44 141; the biotin fragment was amplified between positions 4557 and 5947 of the pTYB4 vector and digested by NotI at 5748 . PCR products were purified on spin columns (BD Chroma Spin 1000 or 100), and fragment ligation (T4 DNA ligase, New England Biolabs) was conducted with excess multidigoxigenin and multibiotin fragments to 20 
to ensure optimal reaction of most of the central fragments. Ligation products were then purified and selected through gel extraction (QiaQUICK Gel Extraction Kit, QIAGEN). The final products were unnicked DNA molecules of, respectively, $5.42 \pm 0.03$ and $4.06 \pm 0.23 \mu \mathrm{m}$ with multiple biotin labels on one end and multiple digoxigenin labels on the other end.

\section{Microfluidic setup}

A polydimethylsiloxane (PDMS) microchannel $(2 \mathrm{~cm} \times$ $2 \mathrm{~mm} \times 110 \mu \mathrm{m})$ was placed on a glass coverslip of $24 \times$ $40 \mathrm{~mm}$ (Erie Scientific Company, France) treated with Sigmacote (Sigma-Aldrich) followed by antidigoxigenin (Roche, France) for subsequent binding of digoxigenin-labeled DNA molecules. To minimize adsorption of Rad51 onto the glass surface and onto the PDMS walls, the channel was coated with Pluronic F-127 (Sigma-Aldrich).

The biotin-labeled ends of DNA molecules were bound to streptavidin-coated $2.8-\mu \mathrm{m}$ magnetic beads (Dynabeads M-280 Streptavidin) in a binding buffer [10 mM tris(hydroxymethyl)aminomethane hydrochloride (Tris-HCl), $\mathrm{pH} 7.5,1 \mathrm{mM}$ ethylenediamine tetra-acetic acid (EDTA), $50 \mathrm{mM} \mathrm{NaCl}$. The DNA-bound bead suspension was then introduced at a controlled flow rate into the PDMS microchannel. After $30 \mathrm{~min}$ of incubation, most of the unbound beads were washed out of the channel with TE buffer (10 mM Tris-HCl, 1 mM EDTA, pH 7.5).

\section{RESULTS}

To explain the specificity of HR, we introduced the two aforementioned hypotheses.

(i) The NPF stretched-to-compact $(S \rightarrow C)$ transition is driven by the torque developed during the interaction between the two DNA molecules.

(ii) The $S \rightarrow C$ transition favors the ADP-bound state and the consequent NPF dismantling.

These hypotheses challenge the historical picture, which considers ATP hydrolysis to be the cause of the $S \rightarrow C$ transition [20-22]. To verify them, we assembled the NPF on a single DNA molecule inside a microfluidic channel, where the concentration of reactants (Rad51 proteins, nucleotides, and cofactors) can be modified at will. We used hybrid magnetic tweezers [HMT, Fig. 1(a)] to constantly monitor the length and the twist of the NPF and to impose a given tension and torque to it. The length and the twist of the NPF were normalized to that of B-DNA. In practice, the canonical active form of $\operatorname{NPF}$ ( $S$ state) has a normalized extension of $L / L_{0}=$ 1.5 and a supercoiling degree of $\sigma=-0.43$, like the common standard in the literature $[9,23]$.

Similarly to other previous work [15,24], the NPFs were assembled onto a double-stranded DNA (dsDNA), because single-stranded DNA (ssDNA) does not store torsional energy. Although Rad51 polymerizes on ssDNA in vivo, it has been shown that polymerization also occurs on dsDNA, with the same structure and biochemical activity [9,25]. Moreover, since ATP is not hydrolyzed during the homology search [6], the reaction remains reversible during this phase. The conservation of energy implies that the order by which the ssDNA and the dsDNA interact with the NPF does not change the free energy between the beginning of the process and the formation of a three-strand synapse.

The ATPase activity of the Rad51 protein was controlled by changing the ionic conditions, ATP hydrolysis being prevented in the presence of $\mathrm{Ca}^{2+}$ and permitted in the presence of $\mathrm{Mg}^{2+}[26,27]$.

\section{A. The compact NFP and the compact-stretched transition}

We first verified the existence of a compact NFP. The Rad51 protein was injected in the observation chamber, together with ATP and $\mathrm{Ca}^{2+}$, while the dsDNA molecule was kept under tension (2-3 pN) and with a supercoiling degree locked at $\sigma=0$. In the presence of $\operatorname{Rad} 51$, the DNA only slightly lengthened [Fig. 1(b), $t=10-20 \mathrm{~min}$ ]. Then, the molecule was unwound in a stepwise manner [dotted line in Fig. 1(b)]. As expected, the NPF length increased consequently (continuous line) and reached the canonical values of $L / L_{0}=1.5$ at $\sigma=-0.43(t=60 \mathrm{~min})$.

To rule out the hypothesis that the Rad51 protein progressively binds to the dsDNA molecule during DNA unwinding, we repeated the experiment with the Rad51 protein transiently injected into the chamber, but washed before DNA unwinding. The results were identical and are summarized in Fig. 2(a), where the NPF length is plotted as a function of the supercoiling degree. This proves that, in the absence of ATP hydrolysis, Rad51 stoichiometrically binds to the dsDNA, even when kept locked at $\sigma=0$. This produces a compact NPF, whose length is dictated by the DNA molecule extension at rest.

\section{B. Stretched-compact transition depends on ATPase activity}

Although the NPF can be assembled in the compact state at $\sigma=0$ and then driven to the stretched conformation through a progressive unwinding, the contrary is not true. When assembled on a DNA molecule previously unwound to $\sigma=-0.43$, the NPF reached directly its canonical length of $L / L_{0}=$ 1.5. Under ATPase-permitting conditions, the NPF shortened under positive supercoiling to reach $L / L_{0} \simeq 1$ at $\sigma=0$ [Fig. 2(b), $\mathbf{\Delta}$ ]. Conversely, if ATP hydrolysis was impeded, the NPF only moderately shortened under positive twisting $(\sigma \rightarrow 0)$ and never reached the compact state [Fig. 2(b), o]. Thus, the $S \rightarrow C$ transition does not occur without ATP hydrolysis. Interestingly, as soon as $\mathrm{Ca}^{2+}$ ions were replaced by $\mathrm{Mg}^{2+}$ ions, the nucleoprotein filament spontaneously shortened [Fig. 2(c), bottom line, red].

This behavior was not only the result of Rad51 depolymerization because when the filament was unwound back it partially extended [Fig. 2(a), $\diamond$ and $\boldsymbol{\Delta}$ ]. In addition, the NPF shortening was several times slower on nicked dsDNA molecules [Fig. 2(c), top line, black], where no torsion was applied. This shrinkage cannot be due to plectoneme formation either, because the rate of DNA-plectoneme folding and unfolding at these forces $(1-3 \mathrm{pN})$ as a function of the supercoiling degree [28] is almost double the value observed here. Instead, the plateau of the final part of the unwinding [Fig. 2(a), $\diamond$ and $\boldsymbol{\Delta}$ ] likely corresponds to the torsional behavior of naked DNA at these forces [28], which indicates partial depolymerization during twisting. This suggests that the NPF could be driven to a more compact state by a 

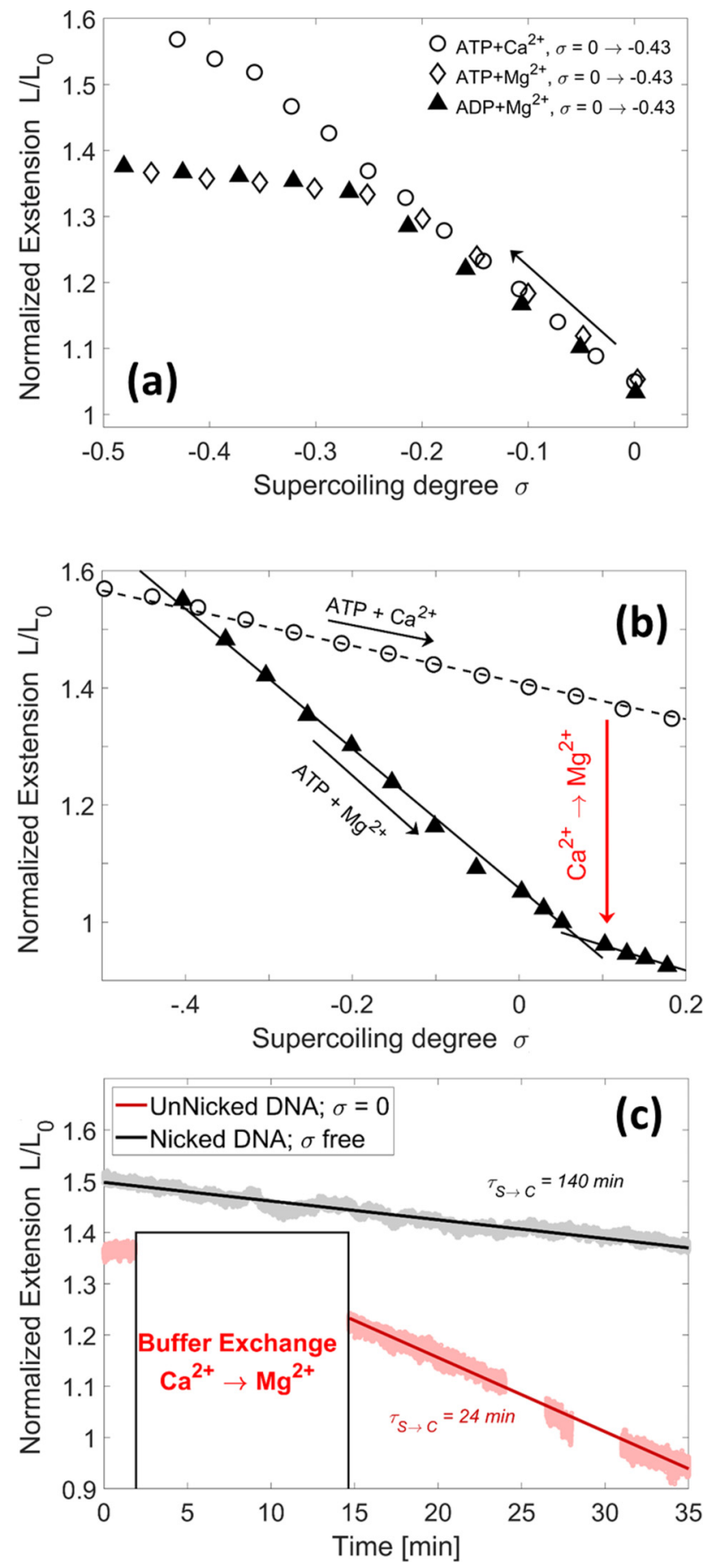

FIG. 2. Structural transition of the NPF. (a) Elongation of the NPF under negative supercoiling, with no Rad51 protein in the solution. In $\mathrm{Ca}^{2+}$ buffer the NPF reaches the canonical length of the active filament $(\circ)$, while in $\mathrm{ATP}+\mathrm{Mg}^{2+}(\diamond)$ or $\mathrm{ADP}(\boldsymbol{\Delta})$ it reaches an intermediate extension. (b) Compaction of the NPF under positive supercoiling, in $\mathrm{Mg}^{2+}$ buffer (ATPase-permitting conditions; $\boldsymbol{\Lambda}$ ) and in $\mathrm{Ca}^{2+}$ buffer (ATPase-nonpermitting conditions; $\circ$ ). The NPF only moderately shortens in $\mathrm{Ca}^{2+}$ buffer, with a length of $L / L_{0} \simeq 1.4$ at $\sigma=0$. When $\mathrm{Ca}^{2+}$ is replaced by $\mathrm{Mg}^{2+}$ [red (gray) arrow and panel (c)], the NPF progressively evolves toward the compact state (bottom curve, red). NPF shortening is also observed on nicked dsDNA, where the torsion is not imposed (top curve, black). However, the shortening rate is fivefold smaller than when the NPF is forced to $\sigma=0$. torsional constraint in a partially reversible manner, but part of the protein depolymerizes during twisting under ATPasepermitting conditions [20]. The fact that this NPF compaction cannot be induced with no ATPase activity also suggests a potential mechanochemical coupling between ATP hydrolysis and the conformational state of the NPF, as already proposed in previous works $[15,16,27,29]$. Moreover, the high torsional rigidity that we observed under ATPase-inhibiting conditions confirms recent results on Rad51-dsDNA mechanics [15] and Rad51-ssDNA structure [27].

With these experiments, we show the following.

(i) The NPF exists in two states $(C$ and $S)$, with the same number of Rad51 proteins bound.

(ii) The stretched NPF is stable under ATPase-inhibiting conditions, whereas the compact one requires ATP hydrolysis.

(iii) The $S \rightarrow C$ transition can be mechanically induced by torsion of the dsDNA within the NPF, under ATPasepermitting conditions.

\section{Energy landscape of the stretched-compact transition}

We used our results along with the previous literature to estimate the energy difference between the stretched and the compact state of the NPF, the torsional stiffness of the NPF, and the energy barrier between the two states.

We modeled the NPF as a polymer made of $N \operatorname{Rad51}$ monomers, among which $n_{s}$ are in the $S$ state and $N-n_{s}$ are in the $C$ state. When perturbed by torsion or traction, the monomers either deform elastically or switch from one state to the other $(S \rightleftharpoons C)$. Given the small nominal strain per monomer in the NPF, we assume elastic response with torsional and longitudinal stiffnesses $K_{\vartheta}$ and $K_{\ell}$ per monomer, respectively. Eventually, we neglect the protein chemical potential and binding energy, as at negative supercoiling depolymerization is much slower than the $S \rightleftharpoons C$ transition $[12,16,17]$ The free energy $G$ is thus the sum of the following:

(i) the elastic energy stored in the strained NPF (extension $L$ and torsion $\Theta$ ), $\Gamma)$,

(ii) the work injected by the tweezers (force $F$ and torque

(iii) the internal energy difference $\Delta \varepsilon$ between the two conformations, and

(iv) the entropic contribution due to the mixing of monomers in the $S$ state and the C state:

$$
\begin{aligned}
G\left(L, \Theta, n_{s}\right)= & \overbrace{\frac{K_{l}}{2 N}\left\{L-\left[N \ell_{s}-\left(N-n_{s}\right) \Delta \ell\right]\right\}^{2}}^{\text {Elastic Energy from Extension }} \\
& +\overbrace{\frac{K_{\vartheta}}{2 N}\left\{\Theta-\left[N \vartheta_{s}+\left(N-n_{s}\right) \Delta \vartheta\right]\right\}^{2}}^{\text {Elastic Energy from Torsion }} \\
& -\underbrace{(F L+\Gamma \Theta)}_{\text {Tweezers Work }}+\underbrace{n_{s} \Delta \varepsilon}_{\text {Conformational Energy }} \\
& +\underbrace{N k_{B} T\left[\frac{n_{s}}{N} \ln \frac{n_{s}}{N}+\left(1-\frac{n_{s}}{N}\right) \ln \left(1-\frac{n_{s}}{N}\right)\right]}_{\text {Entropy }} .
\end{aligned}
$$


TABLE I. Parameters of the two-state model.

\begin{tabular}{lcc}
\hline \hline Parameter & Value & Reference \\
\hline$K_{\ell}$ & $350 \mathrm{pN} / \mathrm{nm}$ & {$[30]$} \\
$\boldsymbol{K}_{\vartheta}$ & To be determined & Fitted \\
$\vartheta_{s}$ & $1.082 \mathrm{rad}$ & {$[15]$} \\
$\Delta \vartheta$ & $0.803 \mathrm{rad}$ & {$[15]$} \\
$\ell_{s}$ & $1.55 \mathrm{~nm}$ & {$[9]$} \\
$\Delta \ell$ & $0.53 \mathrm{~nm}$ & {$[9]$} \\
$\Delta \varepsilon$ & To be determined & Fitted \\
$F$ & $0-10 \mathrm{pN}$ & Measured \\
$L$ & $0-8 \mu \mathrm{m}$ & Measured \\
$\Gamma$ & $0-30 \mathrm{pN} \mathrm{nm}$ & Measured \\
$\Theta$ & $0-10000 \mathrm{rad}$ & Measured \\
\hline \hline
\end{tabular}

In Eq. (1), $\ell_{s}$ and $\vartheta_{s}$ are respectively the length and the twist per unit of $\operatorname{Rad} 51$ in the $S$ state, and $\Delta \ell$ and $\Delta \vartheta$ are the respective differences in the $C$ state. All the parameters are summarized in Table I.

At the equilibrium, the free energy is minimal. Thus, all partial derivatives $\partial G / \partial n_{s}, \partial G / \partial L$, and $\partial G / \partial \Theta$ vanish (see details in Appendix A):

$$
\begin{aligned}
& \frac{n_{s}}{N}=\frac{1}{1+e^{(\Gamma \Delta \vartheta-F \Delta \ell+\Delta \varepsilon) / k_{B} T},} \\
& \frac{L}{N}=\frac{F}{K_{\ell}}+\ell_{s}-\left(1-\frac{n_{s}}{N}\right) \Delta \ell, \\
& \frac{\Theta}{N}=\frac{\Gamma}{K_{\vartheta}}+\vartheta_{s}+\left(1-\frac{n_{s}}{N}\right) \Delta \vartheta .
\end{aligned}
$$

By combining Eqs. (2) and (4), we deduce the analytical function of the supercoiling degree $\sigma$ of the NPF as a function of the torque $\Gamma$ :

$$
\sigma(\Gamma)=\frac{\Theta}{N \vartheta_{0}}-1=\frac{1}{\vartheta_{0}}\left[\frac{\Gamma}{K_{\vartheta}}-\frac{\Delta \vartheta}{1+e^{(\Gamma \Delta \vartheta-F \Delta \ell+\Delta \varepsilon) / k_{B} T}}\right],
$$

with $\vartheta_{0}$ being the canonical torsion of dsDNA.

Equation (5) indicates that there is a critical torque,

$$
\Gamma^{*}=\frac{F \Delta \ell-\Delta \varepsilon}{\Delta \vartheta},
$$

at which the mechanical energy introduced by the tweezers exactly compensates the energy difference of the transition $\Delta \varepsilon$. From this perspective, the NPF is a mechanosensitive complex, with maximal sensitivity at $\Gamma^{*}$ : for a little perturbation of the torque around $\Gamma^{*}$, the portion of $\operatorname{Rad} 51$ proteins in the $S$ state varies very quickly and undergoes the transition to the $C$ state for $\Gamma>\Gamma^{*}$.

\section{Mechanical and energetic properties of the transition}

To test the prediction of Eq. (5), we used the HMT to measure the torque developed by the NPF as a function of its supercoiling degree. Following the method developed by Bryant et al. [31], we deduced the torque from the angular velocity $\omega$ of the bead in the absence of external angular constraints. This condition is obtained when the side magnets are lifted, and it corresponds to the continuous segments
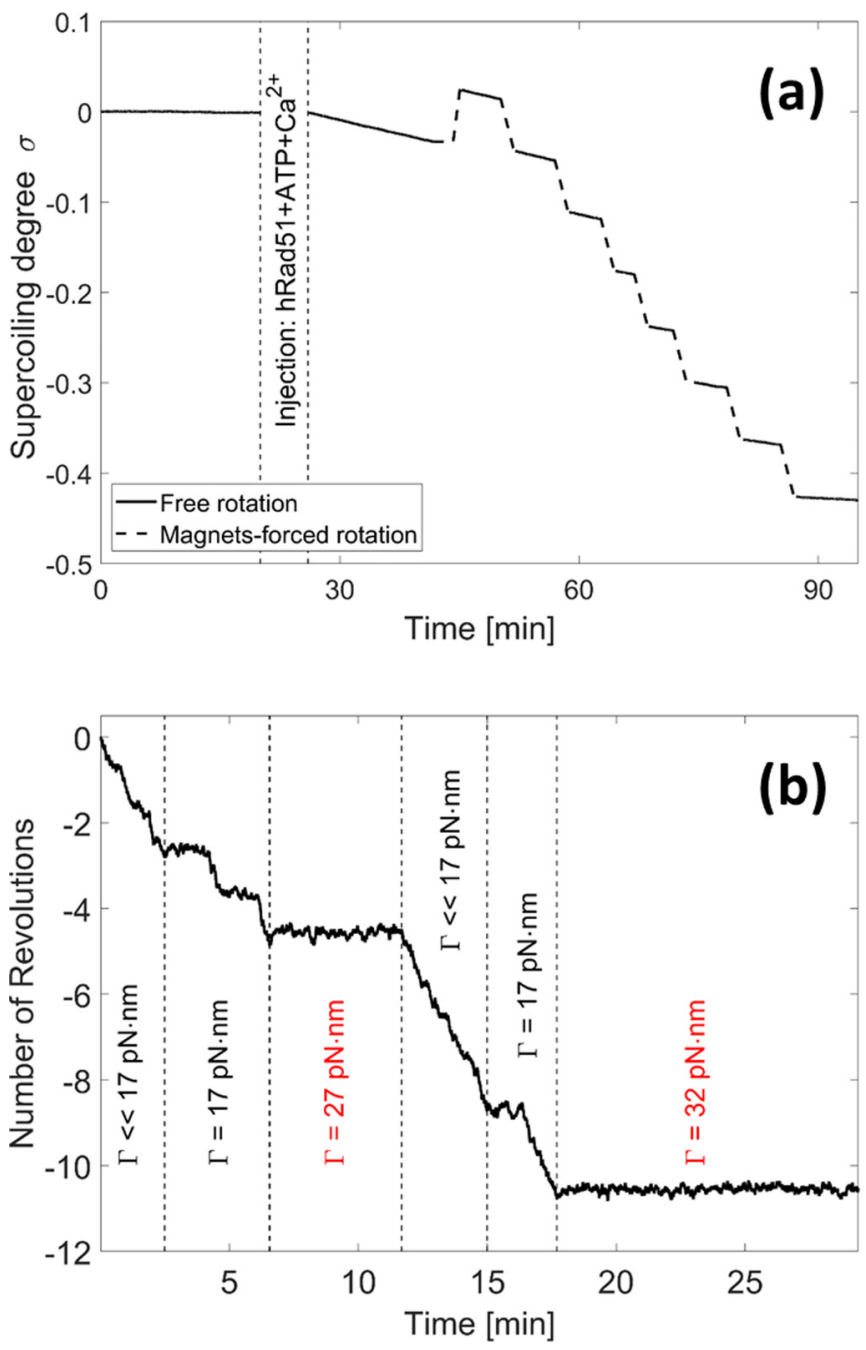

FIG. 3. Torque measurements. (a) Rotation of the nucleoprotein filament as a function of time, at different supercoiling degrees. The residual horizontal magnetic field was nullified during free rotation (continuous segments). An external torque was applied to change the supercoiling degree of the filament (dashed line). During filamentinduced rotation, the tweezers applied a constant pulling force of $F=1.4$ pN. (b) Direct measurement of the critical torque $\Gamma^{*}$. For very small magnetic torques (nonmeasurable), the filament carries the bead. The stronger the torque is, the more laborious the rotation becomes, up to a point where it is completely stalled $(\Gamma>17 \mathrm{pN}$ $\mathrm{nm})$.

in Fig. 3(a). The torque is indeed $\Gamma=\xi \omega$, where $\xi$ is the rotational drag coefficient directly inferred from the angular fluctuation of the bead. Due to its large rotational drag, the bead rotates slowly. Thus, we used the side magnets to rapidly unwind the NPF [dashed segments in Fig. 3(a)] and to measure the angular velocity $\omega(\sigma)$ at different supercoiling degrees. Experiments were replicated 24 times under ATPasepermitting conditions $\left(\mathrm{Mg}^{2+}\right)$ and 26 times under ATPaseinhibiting conditions $\left(\mathrm{Ca}^{2+}\right)$.

We also used the HMT to apply a calibrated torque during the NPF revolution and thus to directly measure the stall torque. We observed that for $\Gamma \ll 17 \mathrm{pN} \mathrm{nm}$ the NPF unwound freely, while larger torques $(\Gamma>17 \mathrm{pN} \mathrm{nm})$ blocked 

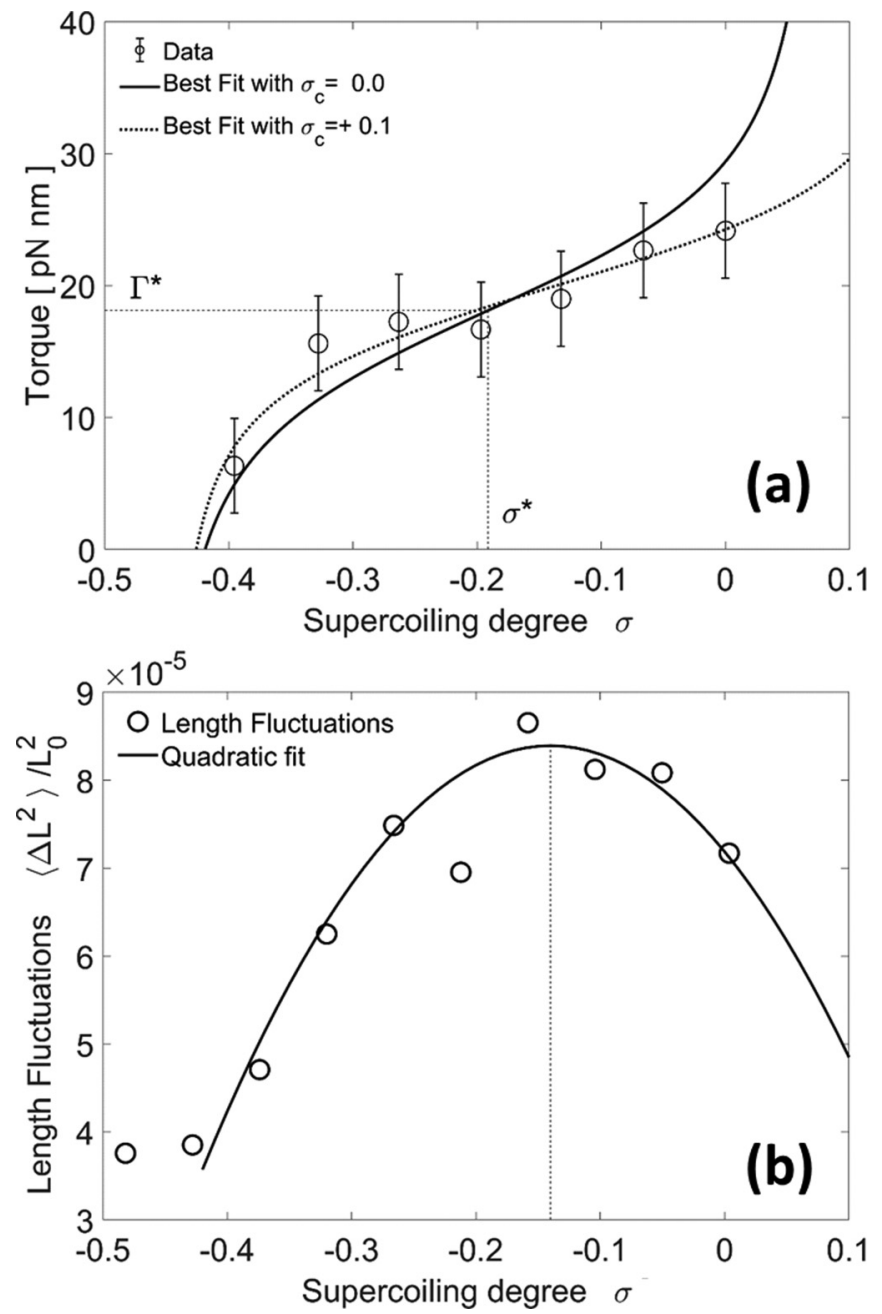

FIG. 4. Torque and supercoiling degree. (a) Torque developed by the NPF as a function of the supercoiling degree imposed by the HMT. The solid line indicates a fit following Eq. (5). The critical torque $\Gamma^{*}$ was deduced from Eq. (6), and its corresponding supercoiling degree was computed from Eq. (5). Error bars represent the standard deviation obtained from different experiments. (b) Fluctuations of the NPF relative length $\left\langle\Delta L^{2}(\sigma)\right\rangle$ as a function of the supercoiling degree. The continuous line represents the best quadratic fit for the fluctuations expected using a two-state model. The best agreement is obtained for a slightly positive supercoiling of the compact state $\sigma_{c}=+0.14$. A value of $\sigma_{c}=+0.1$ clearly optimizes the fit of $\Gamma(\sigma)$ [dotted line in panel (a)].

the bead revolution [Fig. 3(b)]. An intermediate regime appeared at $\Gamma \simeq 17 \mathrm{pN} \mathrm{nm}$, where the unwinding phases were intercalated by pauses. This suggests the existence of a critical torque $\Gamma^{*}=17 \pm 5 \mathrm{pN} \mathrm{nm}$ (the error bar corresponds to the standard deviation obtained from 12 measurements).

The torque corresponding to each supercoiling degree is reported in Fig. 4(a). Here, the torque-supercoiling curve $\Gamma(\sigma)$ was fitted to Eq. (5) to determine the energy difference $\Delta \varepsilon$ between the $S$ state and the $C$ state and the torsional stiffness $K_{\vartheta}$. The best agreement was obtained for $\Delta \varepsilon=-14 \pm 2 \mathrm{pN}$ $\mathrm{nm}$ and $K_{\vartheta}=390 \pm 110 \mathrm{pN} \mathrm{nm}$. With a stretching force of $F=2 \mathrm{pN}$, we expect a critical torque of $\Gamma^{*}=18 \pm 2 \mathrm{pN} \mathrm{nm}$ for a critical supercoiling degree of $\sigma^{*}=-0.19 \pm 0.03$. This value is consistent with the experimental value of $17 \mathrm{pN} \mathrm{nm}$ deduced from Fig. 3(b).

\section{E. NPF length fluctuations}

An additional prediction of the two-state model is that the fluctuations of the NPF length have to be maximal at the critical supercoiling degree $\sigma^{*}$, where $n_{s}=N / 2$, and minimal when $n_{s}=0$ or $n_{s}=N$ [see Eq. (B2)]. To verify the prediction, we measured the variance of the NPF length $\left\langle\Delta L^{2}\right\rangle$ at different $\sigma$ [Fig. 4(b)].

As expected, around $\sigma^{*}$ the variance is significantly larger than at $\sigma=-0.43$, where the $S \rightleftharpoons C$ transition does not contribute to the fluctuations. Hereafter denoted $\left\langle\Delta L_{S \rightleftharpoons C}^{2}\right\rangle / L_{0}^{2}$, this difference in amplitude is precisely predicted by the binomial distribution (see details in Appendix B). For our DNA molecule constituted by 15941 base pairs (roughly 5300 Rad51 molecules) we expect $\left\langle\Delta L_{S \rightleftharpoons C}^{2}\right\rangle / L_{0}^{2}=1.2 \times 10^{-5}$. Experimentally, we measured $\left\langle\Delta L_{S \rightleftharpoons C}^{2}\right\rangle / L_{0}^{2}=(4.8 \pm 1) \times 10^{-5}$ [Fig. 4(b)]. This discrepancy can be understood if one admits that there is a certain degree of cooperativity between adjacent Rad51 proteins, during the $\mathrm{S} \rightleftharpoons \mathrm{C}$ transition. In this case, the NPF would be constituted by fewer but larger active units, which would result in larger fluctuations. From the measured fluctuation amplitude and using Eq. (B4), we estimate that the active unit is made of approximately four Rad51 monomers (Hill index $=4 \pm 1$ ), which simultaneously undergo the $S \rightleftharpoons$ $C$ transition. Noticeably, this observation is compatible with the fact that Rad51 also cooperatively polymerizes and/or depolymerizes on the DNA to form the NPF, with a Hill Index of about $4.5[20,32,33]$.

The quadratic fit in Fig. 4(a) also suggests that the compact conformation of the NPF is shifted toward positive supercoiling, around $\sigma_{c}=+0.14$. Unfortunately, neither the torque nor the fluctuations were measurable at positive supercoiling, where the NPF rapidly depolymerized. However, when data in Fig. 4(a) were fitted with $\vartheta_{c}$ as an additional free parameter, we obtained the best agreement for $\vartheta_{c}$ corresponding to $\sigma=$ +0.1 and $\Delta \varepsilon=-20 \pm 2 \mathrm{pN} \mathrm{nm}$. Interestingly, $\sigma=+0.1$ for the compact state is also compatible with the data shown in Fig. 2(b), where the curve $L(\sigma)$ exhibits a change of slope at $\sigma \geqslant 0.05$. In the following discussion, we use $\Delta \varepsilon \simeq-20 \mathrm{pN}$ $\mathrm{nm}$.

\section{F. Energy landscape}

Figure 5(a) shows what the energy landscape of the $S \rightarrow C$ transition looks like, using the two-state model described by Eq. (1) and the values of $\Delta \varepsilon$ and $K_{\vartheta}$ determined experimentally. A clear energy barrier of $\Delta G \simeq 60 \mathrm{pN} \mathrm{nm}$ appears between the two states.

Using Eq. (2), we also estimated the probability $n_{s} / N$ of finding a Rad51 monomer in the stretched conformation, as a function of the external work done on the NPF [Fig. 5(b)]. As expected, the probability curve exhibits the maximum steepness where the mechanical work brought by the tweezers at the critical torque $\Gamma^{*}$ (or by base pairing) exactly balances $\Delta \varepsilon$. 
(a)
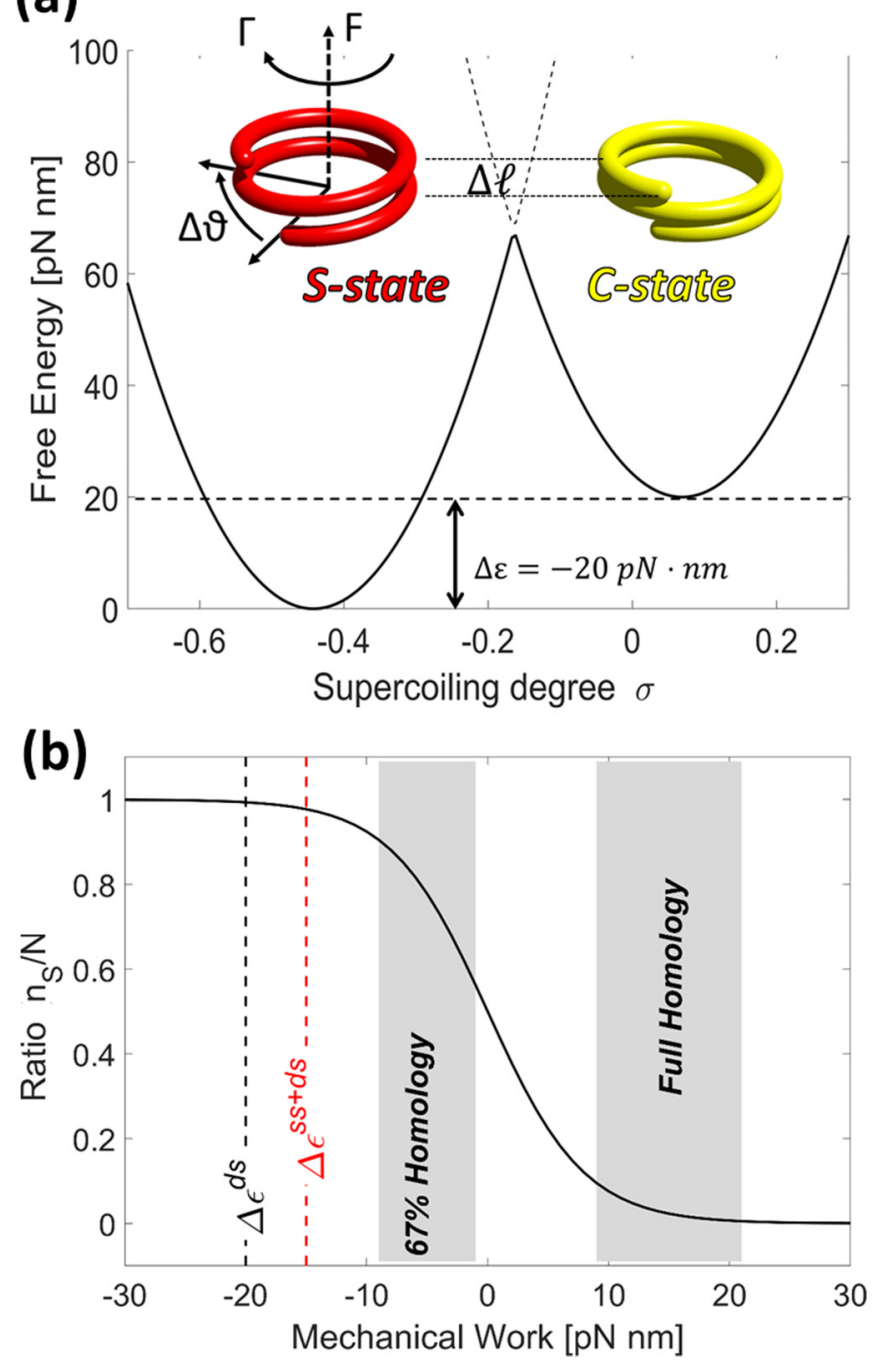

FIG. 5. Energetic properties of the NPF transition. (a) Energy landscape per monomer of $\operatorname{Rad51}$, as a function of the supercoiling degree. (Left) Elastic energy in the stretched conformation. (Right) Elastic energy in the compact conformation. (b) Portion of the NPF in the $S$ state as a function of the mechanical energy supplied by either the tweezers or the DNA molecules [from Eq. (2)]. The gray-shaded areas indicate the energy values when the NPF interacts with either a partially homologous DNA fragment or a fully homologous DNA fragment.

\section{DISCUSSION}

\section{A. Selectivity for homologous sequences}

With these results, we suggest a possible explanation for the reversibility $\rightarrow$ irreversibility transition in HR. As already mentioned, since ATP is not hydrolyzed during the homology search [6], the mechanical energy is conserved and the order by which the ssDNA and the dsDNA interact with the NPF does not affect the energy difference $\Delta \varepsilon^{\text {ss+ds }}$ between the $C$ state and the $S$ state of the NPF with three DNA strands:

$$
\Delta \varepsilon^{\mathrm{ss}+\mathrm{ds}}=\underbrace{\Delta \varepsilon^{\mathrm{ss}}+\Delta W^{\mathrm{ds}}}_{\text {ssDNA first (in vivo) }}=\underbrace{\Delta \varepsilon^{\mathrm{ds}}+\Delta W^{\mathrm{ss}}}_{\text {dsDNA first (experiments) }} .
$$

Here, $\Delta \varepsilon^{\text {ss }}$ and $\Delta \varepsilon^{\text {ds }}$ are the energy differences between the $C$ state and the $S$ state of a NPF, respectively assembled on a ssDNA and on a dsDNA. $\Delta W^{\text {ss }}$ and $\Delta W^{\text {ds }}$ are the mechanical work required to extend, to unwind, and to denature the DNA molecule inside the NPF.

When the NPF was assembled on the dsDNA molecule, we measured an energy difference of $\Delta \varepsilon^{\mathrm{ds}} \simeq-20 \mathrm{pN} \mathrm{nm}$; $\Delta W^{\mathrm{ss}} \simeq+5 \mathrm{pN} \mathrm{nm}$ was estimated by Bustamante et al. [34]. With these values, we computed $\Delta \varepsilon^{\mathrm{ss}+\mathrm{ds}} \simeq-15 \mathrm{pN}$ $\mathrm{nm}$ [dashed line in Fig. 5(b)]. This negative value means that in the three-strand synapse the $S$ state remains favorable as compared to the $C$ state, even considering the additional energy required to extend the third DNA strand (ssDNA), which penalizes the stretched state.

Pairing of a fully homologous sequence brings $8-12 \mathrm{pN}$ $\mathrm{nm}$ per base pair [35,36], i.e., a net energy gain per Rad51 monomer of between 24 and $36 \mathrm{pN} \mathrm{nm}$. Conversely, a denaturation bubble due to a single mismatch in a triplet gives an energy penalty of $\simeq 10 \mathrm{pN} \mathrm{nm} \mathrm{[37],} \mathrm{thus} \mathrm{a} \mathrm{net}$ energy gain per triplet between 6 and $14 \mathrm{pN} \mathrm{nm}$, for a homology of $67 \%$. Those two energy ranges are highlighted in Fig. 5(b). It appears that in the case of partial homology the energy brought by base pairing is not sufficient to fully trigger the $S \rightarrow C$ transition, while the transition occurs with an efficiency of $99 \%$ in the case of full homology.

The observation that the transition cannot be induced mechanically by the tweezers under ATPase-inhibiting conditions [Figs. 2(b) and 2(c)], but it is allowed under ATPasepermitted conditions, indicates that the energy from ATP hydrolysis is stored to keep the NPF in the $S$ state for the reversible phase and dissipated in the mechanically induced transition. This triggers the irreversibility of HR. We also know that hydrolysis does not induce spontaneous dismantling of the NPF $[32,38]$ and that the NPF depolymerization is sensitive to the torque [20]. This suggests that the ATP hydrolysis is not the cause of the $S \rightarrow C$ transition, but it is concomitant with a $S \rightarrow C$ transition triggered by the additional energy brought by the pairing between homologous DNA sequences.

Whereas this hypothesis is unconventional in the recombination context, it is well accepted for the ATP synthase, a mechanoenzyme that bears many similarities with the NPF in terms of both function and structure. The similarities are as follows.

(i) Similarly to the $\gamma$ subunit of ATP synthase, the dsDNA molecule acts as a rotor inside the Rad51 NPF. In both cases, a counterclockwise rotation of a central pivot (i.e., the $\gamma$ subunit or the dsDNA molecule) triggers a change in the chemical state of the complex from ATP to ADP, and vice versa for a clockwise rotation.

(ii) ATP synthase is a ring constituted of three heterodimers. NPF is a helix constituted of three homodimers per turn [39].

(iii) The loops that bind the DNA in recombinase proteins are topologically analogous to those that bind the coiled-coil $\gamma$ subunit in the ATP synthase [40].

(iv) The nucleotide-binding sites of the two proteins are structurally homologous [41]. 
Within this analogy, the proton gradient that fuels the structural change of ATP synthase is comparable to the base pairing within the NPF, which triggers the $S \rightarrow C$ transition.

\section{B. Cooperativity and sensitivity to the length of homologous sequences}

Eventually, the two-state model also captures an additional feature of homologous recombination. HR requires more than 8-15 consecutive homologous base pairs to succeed [7], which is interpreted as a way to avoid recombination in the case of partial homology.

We know that the NPF nucleation requires the simultaneous binding of several $\operatorname{Rad} 51$ proteins, as indicated by a Hill index of $\simeq 4.5[32,33]$. This result is compatible with the hexameric structure of the NPF helix. Similarly, NPF depolymerization occurs by the unbinding of several Rad51 proteins at a time [20]. Eventually, the fluctuations of the NPF length (Sec. IIIE) suggest a certain degree of cooperativity also during the $S \rightleftharpoons C$ transition. All those findings indicate that the active unit of HR may not be the single Rad51, but a complex of four to six proteins.

To explore the potential behavior of a multimeric active unit in the two-state model, we compared the free-energy landscape of a single Rad51 protein (Fig. 6, black line) to that of a Rad51 pentamer [Fig. 6, red (gray) line]. The energy landscape was computed by extrapolating the two-state model to a pentameric structure, without considering any additional term due to the mutual interaction between adjacent Rad51 proteins $[42,43]$. Although qualitative, this extrapolation highlights an interesting feature of the recombinase proteins. Intrinsically more compliant than a single protein, in terms of both torsion and extension, a Rad51 pentamer exhibits multiple little energy barriers $(\simeq 15 \mathrm{pN} \mathrm{nm})$ instead of a large one $(\simeq 60 \mathrm{pN}$ $\mathrm{nm}$ ), in the $S \rightarrow C$ transition. In the case of 15 homologous base pairs, which involve a Rad51 pentamer, the multiple little barriers can be easily overtaken to achieve the $S \rightarrow C$ transition. Conversely, the large barrier that appears in the case of a single homologous triplet slows down the $S \rightarrow C$ transition, thus favoring the unbinding of the nonhomologous sequence and a new search.

\section{CONCLUSION}

In conclusion, using single-molecule measurements and a minimal two-state model, we provided a physically plausible mechanism to explain the sensitivity of homologous recombination to base mismatches and to justify the cooperativity between recombinase proteins. The block diagram in Fig. 7 illustrates the steps of this process, as suggested by our experiments and modeling, along with previous results from the literature.

\section{ACKNOWLEDGMENTS}

We thank H. Arata for conceiving the first experimental setup, A. Renodon-Cornière and M. Takahashi for providing the Rad51 proteins, and T. Strick for his valuable help to produce the DNA fragments. We also warmly thank P. Martin and J. Prost, who carefully read the manuscript and provided critical comments. G.C. thanks Valerio Cappello,
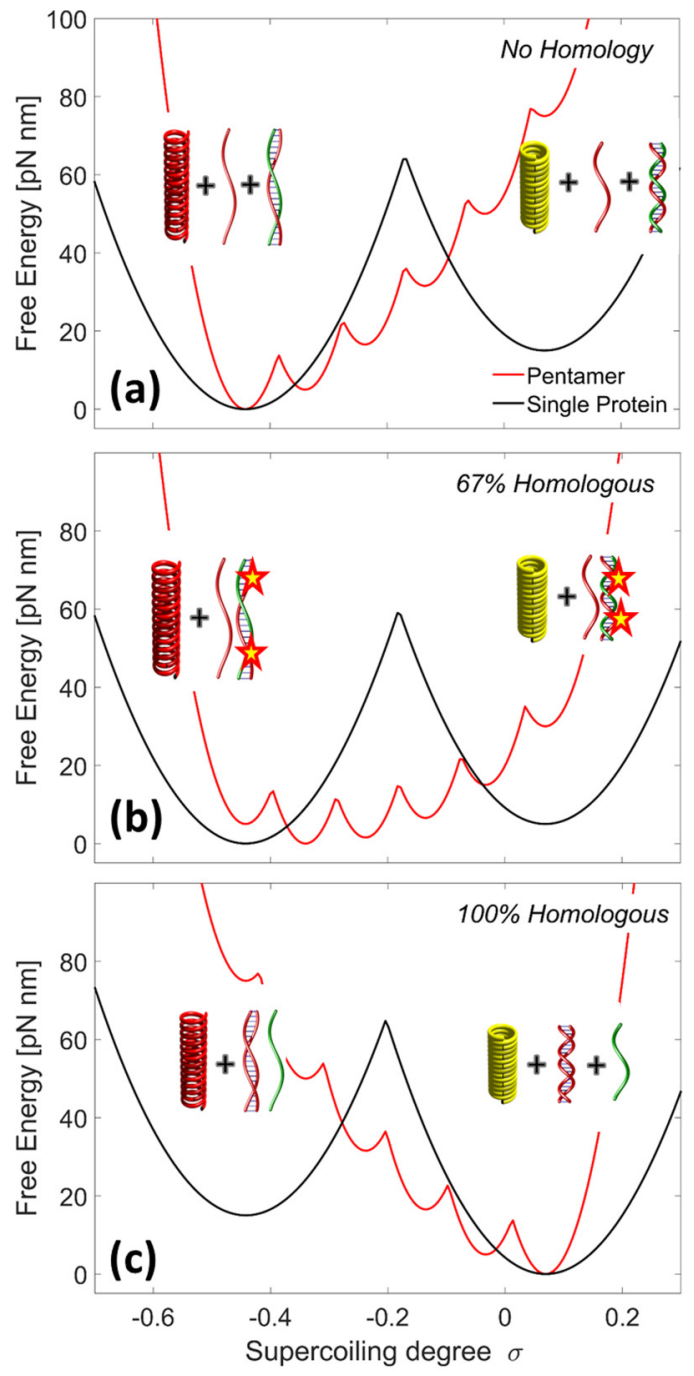

FIG. 6. Energy landscapes of the NPF in the three-strand synapse. Free energy versus supercoiling degree for single (black line) or pentamer [red (gray) line] of Rad51 in the NPF at the beginning of the homology search (a) and in configurations with partial (b) and perfect (c) homology.

with whom he wrote the last chapter of the story. This work was supported by the Agence Nationale de la Recherche (Grant No. ANR-10-BLAN-1013 1-"DynRec"), the Region Ile-de-France in the framework of the Centre de Compétences en Nanosciences en Ile-de-France (Grant No. AI2010-a209_RECOMBINATARGET_CAPPELLO), and the Program "Investissements d'Avenir" (Grants No. ANR-10-LABX-31, No. ANR-10-EQUIPEX-34, and No. ANR-10-IDEX-000102 PSL).

\section{APPENDIX A: FREE ENERGY OF THE ACTIVE TWO-STATE MODEL}

The internal energy of a monomer in the $S$ and $C$ states is given by the contribution of (i) the mechanical energy stored in the monomer (extension and torsion), (ii) the work injected by the tweezers (force and torque), and (iii) the potential 


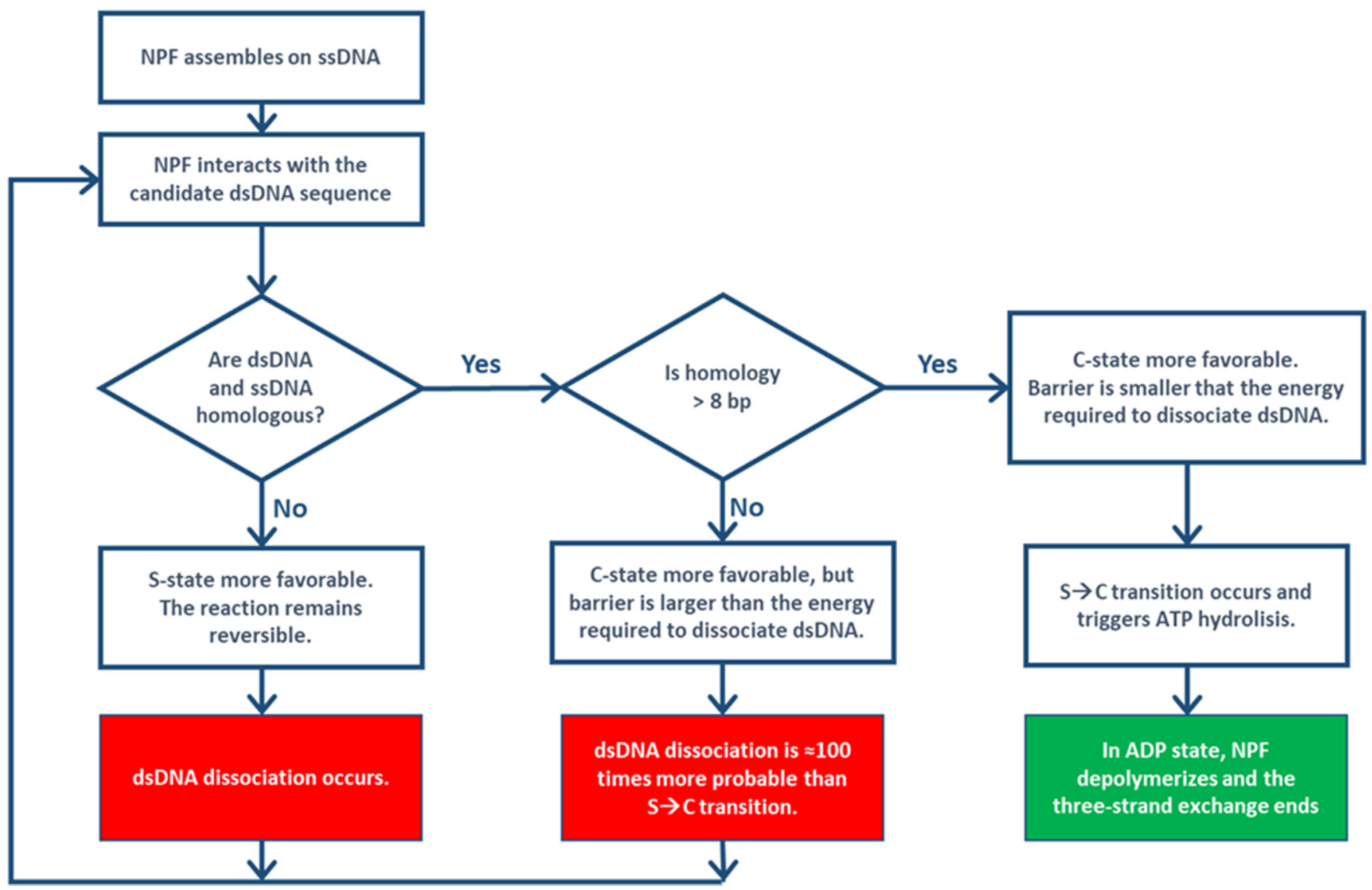

FIG. 7. Block diagram for the homology recognition. Scheme of the potential regulation of the homology search based on the energy properties of the DNA-recombinase assembly.

energy of the state:

$$
\begin{aligned}
U_{S}(\ell, \vartheta)= & \overbrace{\frac{K_{\ell}}{2}\left(\ell-\ell_{s}\right)^{2}}^{\text {Extension Energy }}+\overbrace{\frac{K_{\vartheta}}{2}\left(\vartheta-\vartheta_{s}\right)^{2}}^{\text {Torsion Energy }}-\overbrace{(F \ell+\Gamma \cdot \vartheta)}^{\text {Tweezers Work }}+\varepsilon_{s} \\
U_{C}(\ell, \vartheta)= & \underbrace{\frac{K_{\ell}}{2}\left[\ell-\left(\ell_{s}-\Delta \ell\right)\right]^{2}}_{\text {Extension Energy }}+\underbrace{\left.\frac{K_{\vartheta}}{2}\left[\vartheta-\left(\vartheta_{s}+\Delta \vartheta\right)\right]\right)^{2}}_{\text {Torsion Energy }} \\
& -\underbrace{(F \ell+\Gamma \cdot \vartheta)}_{\text {Tweezers Work }}+\varepsilon_{C} .
\end{aligned}
$$

By summing the internal energy contribution of all the $N$ monomers in series $(U)$, and by considering the contribution from the entropy of mixing $(S)$, one obtains the free energy $G$ of the NPF:

$$
\begin{aligned}
G\left(L, \Theta, n_{s}\right)= & \overbrace{\frac{K_{l}}{2 N}\left\{L-\left[N \ell_{s}-\left(N-n_{s}\right) \Delta \ell\right]\right\}^{2}}^{\text {Elastic Energy from Extension }} \\
& +\overbrace{\frac{K_{\vartheta}}{2 N}\left\{\Theta-\left[N \vartheta_{s}+\left(N-n_{s}\right) \Delta \vartheta\right]\right\}^{2}}^{\text {Elastic Energy from Torsion }}
\end{aligned}
$$

$$
\begin{aligned}
& -\underbrace{(F L+\Gamma \Theta)}_{\text {Tweezers Work }}+\underbrace{n_{s} \Delta \varepsilon}_{\text {Conformational Energy }} \\
& +\underbrace{N k_{B} T\left[\frac{n_{s}}{N} \ln \frac{n_{s}}{N}+\left(1-\frac{n_{s}}{N}\right) \ln \left(1-\frac{n_{s}}{N}\right)\right]}_{\text {Entropy }} .
\end{aligned}
$$

At the equilibrium the free energy is minimal and its derivatives vanish. This results in the following:

$$
\begin{gathered}
\frac{\partial G}{\partial L}=\frac{K_{\ell}}{N}\left\{L-\left[N \ell_{s}-\left(N-n_{s}\right) \Delta \ell\right]\right\}-F=0 \\
\frac{\partial G}{\partial \Theta}=\frac{K_{\vartheta}}{N}\left\{\Theta-\left[N \vartheta+\left(N-n_{s}\right) \Delta \vartheta\right]\right\}-\Gamma=0 \\
\frac{\partial G}{\partial n_{s}}=-F \Delta \ell+\Gamma \Delta \vartheta+\Delta \varepsilon+k_{B} T \ln \frac{n_{s}}{N-n_{s}}=0
\end{gathered}
$$

By solving these equations, one obtains Eqs. (2)-(4).

\section{APPENDIX B: LENGTH FLUCTUATIONS OF THE NUCLEOPROTEIN FILAMENT}

The mean length $(\langle L\rangle)$ of a nucleoprotein filament and its variance $\left(\left\langle\Delta L^{2}\right\rangle\right)$ both depend on the total number of the 
number $n_{s}$ of the Rad51 protein in the $S$ state. According to the binomial distribution, we expect

$$
\begin{gathered}
\frac{\langle L\rangle}{L_{0}}=1+\frac{\Delta \ell}{\ell_{c}} \cdot \frac{n_{s}}{N}, \\
\frac{\left\langle\Delta L^{2}\right\rangle}{L_{0}^{2}}=\frac{1}{N}\left(\frac{\Delta \ell}{\ell_{c}}\right)^{2}\left(\frac{n_{s}}{N}\right)\left(1-\frac{n_{s}}{N}\right),
\end{gathered}
$$

where $\ell_{c}=\ell_{s}-\Delta \ell$ is the length of a $\operatorname{Rad} 51$ protein in the $C$ state.

If we consider that the active unit of $\mathrm{HR}$ is not the single Rad51 protein, but rather a multimeric complex made of $\alpha$ proteins, then the length of each unit is multiplied by $\alpha$, while their number is divided by $\alpha$ ( $\alpha$ is commonly called the Hill index). Whereas the mean length of the molecule remains unchanged [Eq. (B1)], the variance increases proportionally to $\alpha$ :

$$
\left[\frac{\left\langle\Delta L^{2}\right\rangle}{L_{0}^{2}}\right]_{\alpha}=\frac{\alpha}{N}\left(\frac{\Delta \ell}{\ell_{c}}\right)^{2}\left(\frac{n_{s}}{N}\right)\left(1-\frac{n_{s}}{N}\right) .
$$

Due to the $\mathrm{S} \rightleftharpoons \mathrm{C}$ transition, the variance is maximal for $n_{s} / N=1 / 2, \Delta \ell / l_{c}=0.5$ for $\operatorname{Rad} 51$, and the maximal square amplitude of the fluctuations $\left\langle\Delta L_{S \rightleftharpoons C}^{2}\right\rangle$ becomes

$$
\left[\frac{\left\langle\Delta L_{S \rightleftharpoons C}^{2}\right\rangle}{L_{0}^{2}}\right]_{\alpha}=\frac{\alpha}{N} 0.5^{2}\left(\frac{1}{2}\right)\left(\frac{1}{2}\right)=\frac{\alpha}{16 N} .
$$

[1] J. C. Game, Semin. Cancer Biol. 4, 73 (1993).

[2] A. Shinohara, H. Ogawa, and T. Ogawa, Cell 69, 457 (1992).

[3] S. C. Kowalczykowski and A. K. Eggleston, Annu. Rev. Biochem. 63, 991 (1994).

[4] S. C. Kowalczykowski, Annu. Rev. Biophys. Biophys. Chem. 20, 539 (1991).

[5] P. Sung, Science (New York, N.Y.) 265, 1241 (1994).

[6] P. Baumann, F. E. Benson, and S. C. West, Cell 87, 757 (1996).

[7] Z. Qi, S. Redding, J. Y. Lee, B. Gibb, Y. Kwon, H. Niu, W. A. Gaines, P. Sung, and E. C. Greene, Cell 160, 856 (2015).

[8] J. Y. Lee, T. Terakawa, Z. Qi, J. B. Steinfeld, S. Redding, Y. Kwon, W. A. Gaines, W. Zhao, P. Sung, and E. C. Greene, Science (New York, N.Y.) 349, 977 (2015).

[9] X. Yu, S. A. Jacobs, S. C. West, T. Ogawa, and E. H. Egelman, Proc. Natl. Acad. Sci. USA 98, 8419 (2001).

[10] F. E. Benson, A. Stasiak, and S. C. West, EMBO J. 13, 5764 (1994).

[11] I. De Vlaminck, M. T. J. van Loenhout, L. Zweifel, J. den Blanken, K. Hooning, S. Hage, J. Kerssemakers, and C. Dekker, Mol. Cell 46, 616 (2012).

[12] A. Candelli, J. T. Holthausen, M. Depken, I. Brouwer, M. A. M. Franker, M. Marchetti, I. Heller, S. Bernard, E. B. Garcin, M. Modesti, C. Wyman, G. J. L. Wuite, and E. J. G. Peterman, Proc. Natl. Acad. Sci. USA 111, 15090 (2014).

[13] J. C. Bell and S. C. Kowalczykowski, Annu. Rev. Biochem. 85, 193 (2016).

[14] R. Fulconis, A. Bancaud, J.-F. Allemand, V. Croquette, M. Dutreix, and J.-L. Viovy, Biophys. J. 87, 2552 (2004).

[15] M. Lee, J. Lipfert, H. Sanchez, C. Wyman, and N. H. Dekker, Nucleic Acids Res. 41, 7023 (2013).

[16] S. Atwell, L. Disseau, A. A. Z. A. Stasiak, A. A. Z. A. Stasiak, A. Renodon-Cornière, M. Takahashi, J.-L. J.-L. Viovy, and G. Cappello, Nucleic Acids Res. 40, 11769 (2012).

[17] I. Brouwer, T. Moschetti, A. Candelli, E. B. Garcin, M. Modesti, L. Pellegrini, G. J. L. Wuite, and E. J. G. Peterman, EMBO J. 37, e98162 (2018).

[18] J. Lipfert, J. W. J. Kerssemakers, T. Jager, and N. H. Dekker, Nat. Methods 7, 977 (2010).

[19] J. Lipfert, M. Wiggin, J. W. J. Kerssemakers, F. Pedaci, and N. H. Dekker, Nat. Commun. 2, 439 (2011).
[20] J. van Mameren, M. Modesti, R. Kanaar, C. Wyman, E. J. G. Peterman, and G. J. L. Wuite, Nature (London) 457, 745 (2009).

[21] R. B. Robertson, D. N. Moses, Y. Kwon, P. Chan, P. Chi, H. Klein, P. Sung, and E. C. Greene, Proc. Natl. Acad. Sci. USA 106, 12688 (2009).

[22] J. Hilario, A. Ichiro, R. J. Baskin, and S. C. Kowalczykowski, Proc. Natl. Acad. Sci. USA 106, 361 (2007).

[23] A. Stasiak and E. Di Capua, Nature (London) 299, 185 (1982).

[24] A. Reymer, K. Frykholm, K. Morimatsu, M. Takahashi, and B. Nordén, Proc. Natl. Acad. Sci. USA 106, 13248 (2009).

[25] T. Ogawa, X. Yu, A. Shinohara, and E. H. Egelman, Science (New York, N.Y.) 259, 1896 (1993).

[26] D. V. Bugreev and A. V. Mazin, Proc. Natl. Acad. Sci. USA 101, 9988 (2004).

[27] L. H. Fornander, K. Frykholm, A. Reymer, A. RenodonCornière, M. Takahashi, and B. Nordén, Nucleic Acids Res. 40, 4904 (2012).

[28] C. Matek, T. E. Ouldridge, J. P. K. Doye, and A. A. Louis, Sci. Rep. 5, 7655 (2015).

[29] X. Li, X.-P. Zhang, J. A. Solinger, K. Kiianitsa, X. Yu, E. H. Egelman, and W.-D. Heyer, Nucleic Acids Res. 35, 4124 (2007).

[30] J. van Mameren, M. Modesti, R. Kanaar, C. Wyman, G. J. L. Wuite, E. J. G. Peterman, J. van Mameren, M. Modesti, R. Kanaar, C. Wyman, G. J. L. Wuite, and E. J. G. Peterman, Biophys. J. 91, L78 (2006).

[31] Z. Bryant, M. D. Stone, J. Gore, S. B. Smith, N. R. Cozzarelli, and C. Bustamante, Nature (London) 424, 338 (2003).

[32] J. Miné, L. Disseau, M. Takahashi, G. Cappello, M. Dutreix, and J.-L. J.-L. Viovy, Nucleic Acids Res. 35, 7171 (2007).

[33] T. V. D. Heijden, R. Seidel, M. Modesti, R. Kanaar, C. Wyman, and C. Dekker, Nucleic Acids Res. 35, 5646 (2007).

[34] C. Bustamante, Z. Bryant, and S. B. Smith, Nature (London) 421, 423 (2003).

[35] U. Gerland, J. D. Moroz, and T. Hwa, Proc. Natl. Acad. Sci. USA 99, 12015 (2002).

[36] M. Lässig, BMC Bioinf. 8, S7 (2007).

[37] J. SantaLucia and D. Hicks, Annu. Rev. Biophys. Biomol. Struct. 33, 415 (2004).

[38] H.-K. Kim, K. Morimatsu, B. Nordén, M. Ardhammar, and M. Takahashi, Genes cells 7, 1125 (2002). 
[39] A. B. Conway, T. W. Lynch, Y. Zhang, G. S. Fortin, C. W. Fung, L. S. Symington, and P. A. Rice, Nat. Struct. Mol. Biol. 11, 791 (2004).

[40] S. I. Passy, X. Yu, Z. Li, C. M. Radding, J. Y. Masson, S. C. West, and E. H. Egelman, Proc. Natl. Acad. Sci. USA 96, 10684 (1999).
[41] J. P. Abrahams, A. G. Leslie, R. Lutter, and J. E. Walker, Nature (London) 370, 621 (1994).

[42] G. Puglisi and L. Truskinovsky, J. Mech. Phys. Solids 50, 165 (2002).

[43] Y. R. Efendiev and L. Truskinovsky, Continuum Mech. Thermodyn. 22, 679 (2010). 